\title{
On partial sums of normalized Mittag-Leffler functions
}

\author{
Dorina Răducanu
}

\begin{abstract}
This article deals with the ratio of normalized Mittag-Leffler function $\mathbb{E}_{\alpha, \beta}(z)$ and its sequence of partial sums $\left(\mathbb{E}_{\alpha, \beta}\right)_{m}(z)$. Several examples which illustrate the validity of our results are also given.
\end{abstract}

\section{Introduction}

Let $\mathcal{A}$ be the class of functions $f$ normalized by

$$
f(z)=z+\sum_{n=2}^{\infty} a_{n} z^{n}
$$

which are analytic in the open unit disk $\mathcal{U}=\{z \in \mathbb{C}:|z|<1\}$.

Denote by $\mathcal{S}$ the subclass of $\mathcal{A}$ which consists of univalent functions in $\mathcal{U}$.

Consider the function $E_{\alpha}(z)$ defined by

$$
E_{\alpha}(z)=\sum_{n=0}^{\infty} \frac{z^{n}}{\Gamma(\alpha n+1)}, \alpha>0, z \in \mathcal{U},
$$

where $\Gamma(s)$ denotes the familiar Gamma function. This function was introduced by Mittag-Leffler in 1903 [9] and is therefore known as the Mittag-Leffler function.

Key Words: Analytic functions, partial sums, Mittag-Leffler function, univalent function. 2010 Mathematics Subject Classification: 33E12, 30A10.

Received: 01.07.2016

Revised: 15.09 .2016

Accepted: 21.09.2016 
Another function $E_{\alpha, \beta}(z)$, having similar properties to those of MittagLeffler function, was introduced by Wiman [20], [21] and is defined by

$$
E_{\alpha, \beta}(z)=\sum_{n=0}^{\infty} \frac{z^{n}}{\Gamma(\alpha n+\beta)}, \alpha>0, \beta>0, z \in \mathcal{U} .
$$

During the last years the interest in Mittag-Leffler type functions has considerably increased due to their vast potential of applications in applied problems such as fluid flow, electric networks, probability, statistical distribution theory etc. For a detailed account of properties, generalizations and applications of functions (1.2) - (1.3) one may refer to [6], [7], [12], [16].

Geometric properties including starlikeness, convexity and close-to-convexity for the Mittag-Leffler function $E_{\alpha, \beta}(z)$ were recently investigated by Bansal and Prajapat in [1]. Differential subordination results associated with generalized Mittag-Leffler function were also obtained in [14].

The function defined by (1.3) does not belong to the class $\mathcal{A}$. Therefore, we consider the following normalization of the Mittag-Leffler function $E_{\alpha, \beta}(z)$ :

$$
\mathbb{E}_{\alpha, \beta}(z)=\Gamma(\beta) z E_{\alpha, \beta}(z)=z+\sum_{n=1}^{\infty} \frac{\Gamma(\beta)}{\Gamma(\alpha n+\beta)} z^{n+1}, \alpha>0, \beta>0, z \in \mathcal{U} .
$$

Note that some special cases of $\mathbb{E}_{\alpha, \beta}(z)$ are:

$$
\left\{\begin{array}{l}
\mathbb{E}_{2,1}(z)=z \cosh \sqrt{z} \\
\mathbb{E}_{2,2}(z)=\sqrt{z} \sinh (\sqrt{z}) \\
\mathbb{E}_{2,3}(z)=2[\cosh (\sqrt{z})-1] \\
\mathbb{E}_{2,4}(z)=6[\sinh (\sqrt{z})-\sqrt{z}] / \sqrt{z}
\end{array}\right.
$$

Recently, several results related to partial sums of special functions, such as Bessel [10], Struve [22], Lommel [2] and Wright functions [3] were obtained.

Motivated by the work of Bansal and Prajapat [1] and also by the above mentioned results, in this paper we investigate the ratio of normalized MittagLeffler function $\mathbb{E}_{\alpha, \beta}(z)$ defined by (1.4) to its sequence of partial sums

$$
\left\{\begin{array}{l}
\left(\mathbb{E}_{\alpha, \beta}\right)_{0}(z)=z \\
\left(\mathbb{E}_{\alpha, \beta}\right)_{m}(z)=z+\sum_{n=1}^{m} A_{n} z^{n+1}, m \in \mathbb{N}=\{1,2, \ldots\}
\end{array}\right.
$$

where

$$
A_{n}=\frac{\Gamma(\beta)}{\Gamma(\alpha n+\beta)}, \alpha>0, \beta>0, n \in \mathbb{N}
$$


We obtain lower bounds on ratios like

$$
\Re\left\{\frac{\mathbb{E}_{\alpha, \beta}(z)}{\left(\mathbb{E}_{\alpha, \beta}\right)_{m}(z)}\right\}, \Re\left\{\frac{\left(\mathbb{E}_{\alpha, \beta}\right)_{m}(z)}{\mathbb{E}_{\alpha, \beta}(z)}\right\}, \Re\left\{\frac{\mathbb{E}_{\alpha, \beta}^{\prime}(z)}{\left(\mathbb{E}_{\alpha, \beta}\right)_{m}^{\prime}(z)}\right\}, \Re\left\{\frac{\left(\mathbb{E}_{\alpha, \beta}\right)_{m}^{\prime}(z)}{\mathbb{E}_{\alpha, \beta}^{\prime}(z)}\right\}
$$

Several examples will be also given.

Results concerning partial sums of analytic functions may be found in [4], [8], [11], [13], [17], [18], [19].

\section{Main results}

In order to obtain our results we need the following lemma.

Lemma 2.1. Let $\alpha \geq 1$ and $\beta \geq 1$. Then the function $\mathbb{E}_{\alpha, \beta}(z)$ satisfies the next two inequalities:

$$
\begin{gathered}
\left|\mathbb{E}_{\alpha, \beta}(z)\right| \leq \frac{\beta^{2}+\beta+1}{\beta^{2}}, z \in \mathcal{U} \\
\left|\mathbb{E}_{\alpha, \beta}^{\prime}(z)\right| \leq \frac{\beta^{2}+3 \beta+2}{\beta^{2}}, z \in \mathcal{U} .
\end{gathered}
$$

Proof. Under the hypothesis we have $\Gamma(n+\beta) \leq \Gamma(\alpha n+\beta)$ and thus

$$
\frac{\Gamma(\beta)}{\Gamma(\alpha n+\beta)} \leq \frac{\Gamma(\beta)}{\Gamma(n+\beta)}=\frac{1}{(\beta)_{n}}, n \in \mathbb{N},
$$

where

$$
(x)_{n}= \begin{cases}1 & , n=0 \\ x(x+1) \ldots(x+n-1) & , n \in \mathbb{N}\end{cases}
$$

is the well-known Pochhammer symbol.

Note that

$$
(x)_{n}=x(x+1)_{n-1}, \quad n \in \mathbb{N}
$$

and

$$
(x)_{n} \geq x^{n}, \quad n \in \mathbb{N} .
$$

Making use of (2.3) - (2.5) and also of the well-known triangle inequality, for $z \in \mathcal{U}$, we obtain

$$
\left|\mathbb{E}_{\alpha, \beta}(z)\right|=\left|z+\sum_{n=1}^{\infty} \frac{\Gamma(\beta)}{\Gamma(\alpha n+\beta)} z^{n+1}\right| \leq 1+\sum_{n=1}^{\infty} \frac{\Gamma(\beta)}{\Gamma(\alpha n+\beta)} \leq 1+\sum_{n=1}^{\infty} \frac{1}{(\beta)_{n}}
$$




$$
\begin{aligned}
=1+\frac{1}{\beta} \sum_{n=1}^{\infty} \frac{1}{(\beta+1)_{n-1}} \leq 1 & +\frac{1}{\beta} \sum_{n=1}^{\infty} \frac{1}{(\beta+1)^{n-1}}=1+\frac{1}{\beta} \sum_{n=0}^{\infty}\left(\frac{1}{\beta+1}\right)^{n} \\
= & \frac{\beta^{2}+\beta+1}{\beta^{2}}
\end{aligned}
$$

and thus, inequality (2.1) is proved.

Using once more the triangle inequality, for $z \in \mathcal{U}$, we obtain

$$
\left|\mathbb{E}_{\alpha, \beta}^{\prime}(z)\right|=\left|1+\sum_{n=1}^{\infty} \frac{(n+1) \Gamma(\beta)}{\Gamma(\alpha n+\beta)} z^{n}\right| \leq 1+\sum_{n=1}^{\infty} \frac{n \Gamma(\beta)}{\Gamma(\alpha n+\beta)}+\sum_{n=1}^{\infty} \frac{\Gamma(\beta)}{\Gamma(\alpha n+\beta)} .
$$

For $\beta \geq 1$ we have

$$
\frac{n}{(\beta)_{n}}=\frac{n}{\beta(\beta+1)_{n-1}}=\frac{n}{\beta(\beta+1)_{n-2}(\beta+n-1)} \leq \frac{1}{\beta(\beta+1)_{n-2}} .
$$

Taking into account inequalities (2.3) - (2.5) and (2.7), from (2.6), we obtain

$$
\begin{gathered}
\left|\mathbb{E}_{\alpha, \beta}^{\prime}(z)\right| \leq 1+\sum_{n=1}^{\infty} \frac{n}{(\beta)_{n}}+\sum_{n=1}^{\infty} \frac{1}{(\beta)_{n}} \\
\leq 1+\frac{1}{\beta}+\frac{1}{\beta} \sum_{n=2}^{\infty} \frac{1}{(\beta+1)_{n-2}}+\frac{1}{\beta} \sum_{n=1}^{\infty} \frac{1}{(\beta+1)_{n-1}} \\
\leq 1+\frac{1}{\beta}+\frac{1}{\beta} \sum_{n=0}^{\infty}\left(\frac{1}{\beta+1}\right)^{n}+\frac{1}{\beta} \sum_{n=0}^{\infty}\left(\frac{1}{\beta+1}\right)^{n}=\frac{\beta^{2}+3 \beta+2}{\beta^{2}}
\end{gathered}
$$

and thus, inequality (2.2) is also proved.

Let $w(z)$ be an analytic function in $\mathcal{U}$. In the sequel, we will frequently use the following well-known result:

$$
\Re\left\{\frac{1+w(z)}{1-w(z)}\right\}>0, z \in \mathcal{U} \text { if and only if }|w(z)|<1, z \in \mathcal{U} .
$$

Theorem 2.1. Let $\alpha \geq 1$ and $\beta \geq \frac{1+\sqrt{5}}{2}$. Then

$$
\Re\left\{\frac{\mathbb{E}_{\alpha, \beta}(z)}{\left(\mathbb{E}_{\alpha, \beta}\right)_{m}(z)}\right\} \geq \frac{\beta^{2}-\beta-1}{\beta^{2}}, z \in \mathcal{U}
$$

and

$$
\Re\left\{\frac{\left(\mathbb{E}_{\alpha, \beta}\right)_{m}(z)}{\mathbb{E}_{\alpha, \beta}(z)}\right\} \geq \frac{\beta^{2}}{\beta^{2}+\beta+1}, z \in \mathcal{U}
$$


Proof. From inequality (2.1) we get

$$
1+\sum_{n=1}^{\infty} A_{n} \leq \frac{\beta^{2}+\beta+1}{\beta^{2}}, \text { where } A_{n}=\frac{\Gamma(\beta)}{\Gamma(\alpha n+\beta)}, n \in \mathbb{N} .
$$

The last inequality is equivalent to

$$
\frac{\beta^{2}}{\beta+1} \sum_{n=1}^{\infty} A_{n} \leq 1 .
$$

In order to prove the inequality (2.8), we consider the function $w(z)$ defined by

or

$$
\frac{1+w(z)}{1-w(z)}=\frac{\beta^{2}}{\beta+1} \frac{\mathbb{E}_{\alpha, \beta}(z)}{\left(\mathbb{E}_{\alpha, \beta}\right)_{m}(z)}-\frac{\beta^{2}-\beta-1}{\beta+1}
$$

$$
\frac{1+w(z)}{1-w(z)}=\frac{1+\sum_{n=1}^{m} A_{n} z^{n}+\frac{\beta^{2}}{\beta+1} \sum_{n=m+1}^{\infty} A_{n} z^{n}}{1+\sum_{n=1}^{m} A_{n} z^{n}} .
$$

From (2.10), we obtain

$$
w(z)=\frac{\frac{\beta^{2}}{\beta+1} \sum_{n=m+1}^{\infty} A_{n} z^{n}}{2+2 \sum_{n=1}^{m} A_{n} z^{n}+\frac{\beta^{2}}{\beta+1} \sum_{n=m+1}^{\infty} A_{n} z^{n}}
$$

and

$$
|w(z)|<\frac{\frac{\beta^{2}}{\beta+1} \sum_{n=m+1}^{\infty} A_{n}}{2-2 \sum_{n=1}^{m} A_{n}-\frac{\beta^{2}}{\beta+1} \sum_{n=m+1}^{\infty} A_{n}} .
$$

The inequality $|w(z)|<1$ holds true if and only if

$$
\frac{2 \beta^{2}}{\beta+1} \sum_{n=m+1}^{\infty} A_{n} \leq 2-2 \sum_{n=1}^{m} A_{n}
$$

which is equivalent to

$$
\sum_{n=1}^{m} A_{n}+\frac{\beta^{2}}{\beta+1} \sum_{n=m+1}^{\infty} A_{n} \leq 1 .
$$


To prove (2.11), it suffices to show that its left-hand side is bounded above by

$$
\frac{\beta^{2}}{\beta+1} \sum_{n=1}^{\infty} A_{n}
$$

which is equivalent to

$$
\frac{\beta^{2}-\beta-1}{\beta+1} \sum_{n=1}^{m} A_{n} \geq 0
$$

The last inequality holds true for $\beta \geq \frac{1+\sqrt{5}}{2}$.

We use the same method to prove inequality (2.9). Consider the function $w(z)$ given by

$$
\frac{1+w(z)}{1-w(z)}=\frac{\beta^{2}+\beta+1}{\beta+1} \frac{\left(\mathbb{E}_{\alpha, \beta}\right)_{m}(z)}{\mathbb{E}_{\alpha, \beta}(z)}-\frac{\beta^{2}}{\beta+1} .
$$

From the last equality we obtain

$$
w(z)=\frac{-\frac{\beta^{2}+\beta+1}{\beta+1} \sum_{n=m+1}^{\infty} A_{n} z^{n}}{2+2 \sum_{n=1}^{m} A_{n} z^{n}-\frac{\beta^{2}-\beta-1}{\beta+1} \sum_{n=m+1}^{\infty} A_{n} z^{n}}
$$

and

$$
|w(z)|<\frac{\frac{\beta^{2}+\beta+1}{\beta+1} \sum_{n=m+1}^{\infty} A_{n}}{2-2 \sum_{n=1}^{m} A_{n}-\frac{\beta^{2}-\beta-1}{\beta+1} \sum_{n=m+1}^{\infty} A_{n}} .
$$

Then, $|w(z)|<1$ if and only if

$$
\frac{\beta^{2}}{\beta+1} \sum_{n=m+1}^{\infty} A_{n}+\sum_{n=1}^{m} A_{n} \leq 1 .
$$

Since the left-hand side of (2.12) is bounded above by

$$
\frac{\beta^{2}}{\beta+1} \sum_{n=1}^{\infty} A_{n}
$$

we have that the inequality (2.9) holds true. Now, the proof of our theorem is completed. 
In the next theorem we consider ratios involving derivatives.

Theorem 2.2. Let $\alpha \geq 1$ and let $\beta \geq \frac{3+\sqrt{17}}{2}$. Then

$$
\Re\left\{\frac{\mathbb{E}_{\alpha, \beta}^{\prime}(z)}{\left(\mathbb{E}_{\alpha, \beta}\right)_{m}^{\prime}(z)}\right\} \geq \frac{\beta^{2}-3 \beta-2}{\beta^{2}}, z \in \mathcal{U}
$$

and

$$
\Re\left\{\frac{\left(\mathbb{E}_{\alpha, \beta}\right)_{m}^{\prime}(z)}{\mathbb{E}_{\alpha, \beta}^{\prime}(z)}\right\} \geq \frac{\beta^{2}}{\beta^{2}+3 \beta+2}, z \in \mathcal{U} .
$$

Proof. From (2.2) we have

$$
1+\sum_{n=1}^{\infty}(n+1) A_{n} \leq \frac{\beta^{2}+3 \beta+2}{\beta^{2}}, \text { where } A_{n}=\frac{\Gamma(\beta)}{\Gamma(\alpha n+\beta)}, n \in \mathbb{N} .
$$

The above inequality is equivalent to

$$
\frac{\beta^{2}}{3 \beta+2} \sum_{n=1}^{\infty}(n+1) A_{n} \leq 1 .
$$

To prove (2.13), define the function $w(z)$ by

$$
\frac{1+w(z)}{1-w(z)}=\frac{\beta^{2}}{3 \beta+2} \frac{\mathbb{E}_{\alpha, \beta}^{\prime}(z)}{\left(\mathbb{E}_{\alpha, \beta}\right)_{m}^{\prime}(z)}-\frac{\beta^{2}-3 \beta-2}{3 \beta+2}
$$

which gives

$$
w(z)=\frac{\frac{\beta^{2}}{3 \beta+2} \sum_{n=m+1}^{\infty}(n+1) A_{n} z^{n}}{2+2 \sum_{n=1}^{m}(n+1) A_{n} z^{n}+\frac{\beta^{2}}{3 \beta+2} \sum_{n=m+1}^{\infty}(n+1) A_{n} z^{n}}
$$

and

$$
|w(z)|<\frac{\frac{\beta^{2}}{3 \beta+2} \sum_{n=m+1}^{\infty}(n+1) A_{n}}{2-2 \sum_{n=1}^{m}(n+1) A_{n}-\frac{\beta^{2}}{3 \beta+2} \sum_{n=m+1}^{\infty}(n+1) A_{n}} .
$$

The condition $|w(z)|<1$ holds true if and only if

$$
\sum_{n=1}^{m}(n+1) A_{n}+\frac{\beta^{2}}{3 \beta+2} \sum_{n=m+1}^{\infty}(n+1) A_{n} \leq 1 .
$$


The left-hand side of (2.15) is bounded above by

$$
\frac{\beta^{2}}{3 \beta+2} \sum_{n=1}^{\infty}(n+1) A_{n} \quad \text { if } \quad \frac{\beta^{2}-3 \beta-2}{3 \beta+2} \sum_{n=1}^{m}(n+1) A_{n} \geq 0
$$

which holds true for $\beta \geq \frac{3+\sqrt{17}}{2}$.

The proof of (2.14) follows the same pattern. Consider the function $w(z)$ given by

$$
\begin{aligned}
& \frac{1+w(z)}{1-w(z)}=\frac{\beta^{2}+3 \beta+2}{3 \beta+2} \frac{\left(\mathbb{E}_{\alpha, \beta}\right)_{m}^{\prime}(z)}{\mathbb{E}_{\alpha, \beta}^{\prime}(z)}-\frac{\beta^{2}}{3 \beta+2} \\
= & \frac{1+\sum_{n=1}^{m}(n+1) A_{n} z^{n}-\frac{\beta^{2}}{3 \beta+2} \sum_{n=m+1}^{\infty}(n+1) A_{n} z^{n}}{1+\sum_{n=1}^{\infty}(n+1) A_{n} z^{n}} .
\end{aligned}
$$

From (2.16), we can write

$$
w(z)=\frac{-\frac{\beta^{2}+3 \beta+2}{3 \beta+2} \sum_{n=m+1}^{\infty}(n+1) A_{n} z^{n}}{2+2 \sum_{n=1}^{m}(n+1) A_{n} z^{n}-\frac{\beta^{2}-3 \beta-2}{3 \beta+2} \sum_{n=m+1}^{\infty}(n+1) A_{n} z^{n}}
$$

and

$$
|w(z)|<\frac{\frac{\beta^{2}+3 \beta+2}{3 \beta+2} \sum_{n=m+1}^{\infty}(n+1) A_{n}}{2-2 \sum_{n=1}^{m}(n+1) A_{n}-\frac{\beta^{2}-3 \beta-2}{3 \beta+2} \sum_{n=m+1}^{\infty}(n+1) A_{n}} .
$$

The last inequality implies that $|w(z)|<1$ if and only if

$$
\frac{2 \beta^{2}}{3 \beta+2} \sum_{n=m+1}^{\infty}(n+1) A_{n} \leq 2-2 \sum_{n=1}^{m}(n+1) A_{n}
$$

or equivalently

$$
\sum_{n=1}^{m}(n+1) A_{n}+\frac{\beta^{2}}{3 \beta+2} \sum_{n=m+1}^{\infty}(n+1) A_{n} \leq 1 .
$$


It remains to show that the left-hand side of (2.17) is bounded above by

$$
\frac{\beta^{2}}{3 \beta+2} \sum_{n=1}^{\infty}(n+1) A_{n} .
$$

This is equivalent to

$$
\frac{\beta^{2}-3 \beta-2}{3 \beta+2} \sum_{n=1}^{m}(n+1) A_{n} \geq 0 \text { which holds true for } \beta \geq \frac{3+\sqrt{17}}{2} .
$$

Now, the proof of our theorem is completed.

\section{Examples}

In this section we give several examples which illustrate our theorems.

A result involving the functions $\mathbb{E}_{2,2}(z)$ and $\mathbb{E}_{2,3}(z)$, defined by (1.5), can be obtained from Theorem 2.1 by taking $m=0, \alpha=2, \beta=2$ and $m=0, \alpha=$ $2, \beta=3$, respectively.

Corollary 3.1. The following inequalities hold true:

$$
\Re\left\{\frac{\sinh (\sqrt{z})}{\sqrt{z}}\right\} \geq \frac{1}{4}=0,25, \Re\left\{\frac{\sqrt{z}}{\sinh (\sqrt{z})}\right\} \geq \frac{4}{7} \approx 0,57
$$

and

$$
\Re\left\{\frac{\cosh (\sqrt{z})-1}{z}\right\} \geq \frac{5}{18} \approx 0,28, \Re\left\{\frac{z}{\cosh (\sqrt{z})-1}\right\} \geq \frac{18}{13} \approx 1,38 .
$$

Setting $m=0, \alpha=2$ and $\beta=4$ in Theorem 2.1 and Theorem 2.2 respectively, we obtain the next result involving the function $\mathbb{E}_{2,4}(z)$, defined by (1.5), and its derivative.

Corollary 3.2. The following inequalities hold true:

$$
\Re\left\{\frac{\sinh (\sqrt{z})-\sqrt{z}}{z \sqrt{z}}\right\} \geq \frac{11}{96} \approx 0,11, \Re\left\{\frac{z \sqrt{z}}{\sinh (\sqrt{z})-\sqrt{z}}\right\} \geq \frac{32}{7} \approx 4,57
$$

and

$$
\begin{aligned}
& \Re\left\{\frac{\sqrt{z} \cosh (\sqrt{z})-\sinh (\sqrt{z})}{z \sqrt{z}}\right\} \geq \frac{1}{24} \approx 0,04, \\
& \Re\left\{\frac{z \sqrt{z}}{\sqrt{z} \cosh (\sqrt{z})-\sinh (\sqrt{z})}\right\} \geq \frac{8}{5}=1,6 .
\end{aligned}
$$


Remark 3.1. If we consider $m=0$ in inequality (2.13), we obtain $\Re\left\{\mathbb{E}_{\alpha, \beta}^{\prime}(z)\right\}>0$. In view of Noshiro-Warschawski Theorem (see [5]), we have that the normalized Mittag-Leffler function is univalent in $\mathcal{U}$ for $\alpha \geq 1$ and $\beta \geq \frac{3+\sqrt{17}}{2}$.

\section{References}

[1] D. Bansal, J. K. Prajapat, Certain geometric properties of the MittagLeffler functions, Complex Var. Elliptic Equ., 61(3)(2016), 338-350.

[2] M. Çağlar, E. Deniz, Partial sums of the normalized Lommel functions, Math. Inequal. Appl., 18(3)(2015), 1189-1199.

[3] M. Din, M. Raza, N. Yağmur, Partial sums of normalized Wright functions, arXiv:1606.02750v1 [math.CV], 2016.

[4] B. A. Frasin, Generalization of partial sums of certain analytic and univalent functions, Appl. Math. Lett., 21(2008), 735-741.

[5] A. W. Goodman, Univalent functions, vol. I, Mariner Publishing Company, Inc., 1983.

[6] R. Gorenflo, F. Mainardi, S. V. Rogosin, On the generalized Mittag-Leffler type function, Integral Transform. Spec. Funct., 7(1998), 215-224.

[7] I. S. Gupta, L. Debnath, Some properties of the Mittag-Leffler functions, Integral Transform. Spec. Funct., 18(5)(2007), 329-336.

[8] L. J. Liu, S. Owa, On partial sums of the Libera integral operator, J. Math. Anal. Appl., 213(2)(1997), 444-454.

[9] G. M. Mittag-Leffler, Sur la nouvelle fonction $E_{\alpha}(x)$, C. R. Acad. Sci. Paris, 137(1903), 554-558.

[10] H. Orhan, N. Yağmur, Partial sums of generalized Bessel functions, J. Math. Inequal., 8(4)(2014), 863-877.

[11] S. Owa, H. M. Srivastava, N. Saitoh, Partial sums of certain classes of analytic functions, Int. J. Comput. Math., 81(10)(2004), 1239-1256.

[12] T. R. Prabhakar, A singular integral equation with a generalized MittagLeffler function in the kernel, Yokohama Math. J., 19(1997), 7-15.

[13] V. Ravichandran, Geometric properties of partial sums of univalent functions, Math. Newslett., 22(3)(2012), 208-221. 
[14] D. Răducanu, Differential subordinations associated with generalized Mittag-Leffler functions (submitted).

[15] T. O. Salim, Some properties relating to the generalized Mittag-Leffler function, Advances Appl. Math. Anal., 4(1)(2009), 21-30.

[16] A. K. Shukla, J. C. Prajapati, On a generalization of Mittag-Leffler function and its properties, J. Math. Anal. Appl., 337(2007), 797-811.

[17] H. Silverman, Partial sums of starlike and convex functions, J. Math. Anal. Appl., 209(1997), 221-227.

[18] E. M. Silvia, On partial sums of convex functions of order $\alpha$, Houston J. Math., 11(1985), 397-404.

[19] A. E. Tudor, D. Răducanu, On a subclass of analytic functions involving harmonic means, An. Şt. Univ. Ovidius Constanţa, 23(1)(2015), 267-275.

[20] A. Wiman, Über den Fundamental satz in der Theorie der Funcktionen $E_{\alpha}(x)$, Acta Math., 29(1905), 191-201.

[21] A. Wiman, Über die Nullstellun der Funcktionen $E_{\alpha}(x)$, Acta Math., 29(1905), 217-134.

[22] N. Yağmur, H. Orhan, Partial sums of generalized Struve functions, Miskolc. Math. Notes (accepted.)

Dorina RĂDUCANU,

Faculty of Mathematics and Computer Science,

Transilvania University of Braşov,

Bdul Iuliu Maniu 50, 500091 Braşov, Romania.

Email: draducanu@unitbv.ro 